\title{
Utilizando Scratch e Arduino como recursos para o ensino da Matemática
}

\author{
Selma Bessa Sales ${ }^{1}$, Ronald Brasil Silva ${ }^{1}$, Elaine Silva Rocha Sobreira ${ }^{2}$, Marcos \\ Dionisio Ribeiro do Nascimento ${ }^{1}$.
}

${ }^{1}$ Secretaria Municipal da Educação (SME)/ Prefeitura Municipal de Fortaleza (PMF)

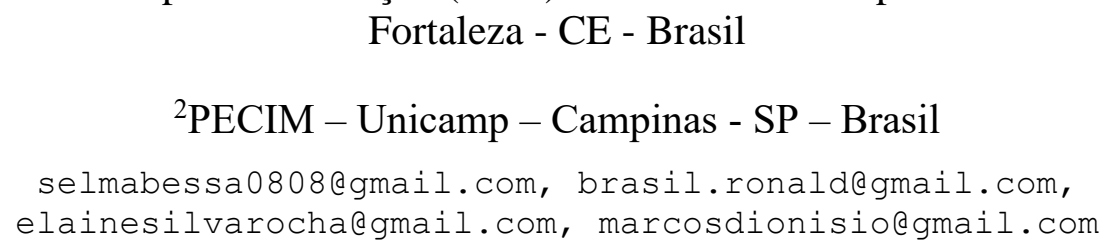

\begin{abstract}
The present article aims to report the experience of using Arduino programmed with Scratch for the development of mathematical contents. It was developed with students of Basic Education, with ages between 11 and 16 years, in one school of the municipal public network of Fortaleza. The central issue involved the following question: Can programming with Scratch and the use of physical prototyping platforms potentiate the teaching of geometry? Faced with this question, we propose a didactic sequence in which students created and program led cubes in mathematics classes, developing learning related to Computational Thinking and geometry. We observed that students were able to interact and learn mathematics successfully, in a programming language environment.
\end{abstract}

Resumo. O presente artigo objetiva relatar a experiência de uso do Arduino programado com Scratch para o desenvolvimento de conteúdos de matemática. Foi desenvolvido com alunos da Educação Básica, com faixa etária entre 11 e 16 anos, em uma escola da rede pública municipal de Fortaleza. A problemática central envolveu a seguinte questão: A programação com Scratch e o uso de plataformas de prototipagem física podem potencializar o interesse dos alunos pelas aulas de geometria? Diante dessa questão, propomos uma sequência didática na qual os alunos criaram e programaram cubos de LED nas aulas de matemática, desenvolvendo aprendizagens relacionadas ao Pensamento Computacional e a geometria. Observamos que os alunos foram capazes de interagir e aprender matemática com exito, em um ambiente de aprendizagem motivador, utilizando a linguagem de programação.

\section{Introdução}

Cada dia mais, as tecnologias digitais estão inseridas nas práticas pedagógicas, buscando a sua transformação. Entendemos que o uso das tecnologias na educação não está relacionado apenas a uma proposta onde o computador passe alguma instrução ao aluno, mas principalmente, em oportunidades para o estudante programar e criar com tecnologias. Sobreira e colaboradores (2016, p. 458) ressaltam a importância de desenvolver atividades que propicie situações para que os alunos "coloquem a "mão-namassa', favorecendo a construção de significados para os fenômenos observados". 
Quando aliada ao currículo escolar, as tecnologias podem transformar o modo de ensinar e aprender, para isso, é necessário rever as práticas pedagógicas que favorecem o uso integrado das tecnologias ao currículo escolar, de modo que as tecnologias não sejam subutilizadas. O potencial das tecnologias pode ser aproveitado através de propostas onde os alunos possam criar e produzir novas tecnologias, ao invés de apenas utilizar tecnologias prontas.

Muitos recursos podem ser utilizados para o desenvolvimento dessa prática, como ambientes de programação por blocos e placas eletrônicas de prototipagem. Um exemplo é o uso do Scratch que permite a programação através de blocos e as placas Arduino, as quais também podem ser programadas através do Scratch, tornando-se acessível para alunos do Ensino Fundamental.

O Scratch é um ambiente de aprendizagem de programação para iniciantes bastante intuitivo, criativo e acessível. Por meio da programação, estimula-se a aprendizagem do pensamento computacional de alunos para resolverem problemas que envolvam conceitos diversos. Criando programações com o Scratch, jovens aprendem a pensar criativamente, a trabalhar de forma colaborativa e a pensar de forma sistemática na solução de problemas. Todas essas criações, podem se tornar ainda mais interativas e concretas, quando aliadas ao uso da placa Arduino.

O Arduino é uma plataforma de prototipagem eletrônica desenvolvida com o "objetivo de criar uma ferramenta fácil para prototipagem rápida, destinado a estudantes sem experiência em eletrônica e programação" [Sobreira et.all, 2016].

De outro lado, a Matemática na Educação Básica deve contemplar uma abordagem curricular integrada, transversal, contextualizada aos componentes curriculares. Na Matemática existem fórmulas prontas ou modalidades didáticas definidas como próprias para serem utilizadas com as questões matemáticas, mas que podem ser aprendidas de forma criativa e significativa quando aliadas às tecnologias digitais.

Dessa forma, o presente trabalho apresenta uma experiência prática envolvendo o uso do Scratch para programar um cubo de LED construído com Arduino, em uma atividade de matemática envolvendo o conteúdo de geometria.

Outros pesquisadores já desenvolveram práticas pedagógicas envolvendo a programação com Arduino na educação básica. Como exemplo temos a experiência de Sobreira e colaboradores (2016, p.456), onde desenvolveram uma prática pedagógica a qual "procurou contribuir para que os estudantes ampliassem seus conhecimentos em relação ao conteúdo de energia através da montagem e programação de circuitos elétricos utilizando (...) placas de prototipagem". Outra experiência é relatada por Amorim e colaboradores (2016, p. 786), os quais desenvolveram uma proposta de construção de humanoides controlados por Arduino, "possibilitando a estudantes do ensino fundamental tornarem-se autores em modelagem, construção e programação usando arquiteturas de hardware e software livre".

Estudos realizados em outros níveis de ensino, como por exemplo em cursos técnicos, também podem ser encontrados, como nos escritos de Albuquerque e colaboradores (2016, p.51) o qual utilizaram o Arduino para introduzir práticas no ensino de linguagem de programação, pois através do Arduino é possível "criar diversas 
situações que auxiliem no aprendizado dos comandos de linguagem, e também estimulem o pensamento computacional".

Considerando e diagnosticando o pouco interesse dos alunos, do colégio onde esse trabalho foi desenvolvido, pelas aulas de geometria, buscamos alternativas para despertar a curiosidade e interesse dos alunos. Compreendendo as possibilidades e o potencial do trabalho com o Arduino e a programação com Scratch, desenvolvemos esse trabalho tendo como principal objetivo, relatar uma prática pedagógica que analisou o desempenho de práticas que exploram o Pensamento Computacional com a utilização de placas de prototipagens como Arduino, programadas com Scratch para 0 desenvolvimento do interesse pelas aulas de geometria.

Essa prática foi desenvolvida em uma escola pública de Ensino Fundamental, em Fortaleza/Ce. Verificou-se que estes recursos permitiram criar um ambiente motivador que proporcionou uma experiência criativa e contextualizada da aprendizagem. Concluímos que os referidos alunos interagiram e aprenderam com as tecnologias, desenvolvendo o aprendizado de matemática num ambiente de linguagem de programação.

Este artigo foi organizado da seguinte forma: a seção 2 apresenta o referencial teórico que embasou nossa prática. A seção 3 descreve a atividade prática desenvolvida com os alunos, enquanto que os resultados e discussões apresentamos na seção 4. Por fim, a seção 5 traz as considerações e as propostas para trabalhos futuros, seguido das referências bibliográficas.

\section{Referencial teórico}

Apesar das tecnologias serem muito utilizadas na educação, ela ainda necessita uma maior integração nas práticas pedagógicas. Muitos estudantes têm mais acesso às tecnologias nos ambientes externos do que é proporcionado no ambiente escolar, gerando um distanciamento entre o ambiente escolar e a realidade social. Buckingham (2007) ressalta a necessidade de os alunos terem um conhecimento crítico dos meios digitais, de forma que as tecnologias digitais não se tornem um mero instrumento de informação.

O construcionismo propõe que o aluno seja protagonista da aprendizagem, entendo os computadores como uma máquia a ser ensinada. Isso é favorecido através do trabalho com linguagem de programação na educação.

Papert, em 1985, já ressaltava a necessidade de se promover o ensino de programação nas escolas, buscando uma aprendizagem mais participativa.

A aprendizagem na escola de hoje não é participativa de modo significativo - e fazer contas não é a imitação de uma atividade excitante e reconhecível da vida adulta. Mas escrever programas para fazer gráficos no computador ou música, ou pilotar uma nave espacial simulada são atividades que têm muitos pontos em comum com as atividades dos adultos. [Papert, 1985, p. 213-214]

Atualmente, essa discussão ainda se faz pertinente, buscando articular a aprendizagem escolar ao cotidiano dos alunos, através de oportunidades onde os alunos possam criar projetos significativos e criativos. Diante dessas necessidades, o MIT desenvolveu em 2007 o Scratch. 
O Scratch consiste em uma linguagem de programação desenvolvida a partir da linguagem LOGO para realizar programações através de blocos de encaixe, facilitando a utilização por crianças. A programação por bloco não exige conhecimentos prévios de programação. Por ser um ambiente aberto, outros desenvolvedores criaram formas de conectar o Scratch ao Arduino, de forma que possibilitasse a programação do Arduino através do Scratch. Algumas opções que utilizamos são: S4A e S2 offline configurado para reconhecer o Arduino através da instalação do pacote HackEduca Conecta oferecido pelo site Hackeduca (http://www.hackeduca.com.br/).

Por sua vez, o Arduino é uma plataforma de prototipagem, destinado a estudantes sem experiência em eletrônica e programação (https://www.arduino.cc/). O Arduino é muito utilizado para o desenvolvimento de computação física, através do uso associado de computação e eletrônica.

Escolhemos a programação com Scratch para programação da placa Arduino, por ser uma linguagem acessível e de fácil compreensão para os alunos. De acordo com Zanetti (2015), com o apoio dos recursos do Scratch, a criança aprende a pensar e a trabalhar de forma criativa e colaborativa.

Conforme as Diretrizes Curriculares para o Ensino Fundamental do Sistema Público Municipal de Ensino de Fortaleza, para que as crianças compreendam os conceitos geométricos, as propriedades de objetos tridimensionais, é sugerido atividades com moldes e planificação de sólidos. Nesse caso as tecnologias constituem importantes recursos para o estudante desenvolver essas relações, ao permitir uma participação mais ativa que favorece a criatividade. Na matemática os alunos podem utilizar os recursos digitais para representar graficamente, processar e transformar dados, visualizar conceitos e aprofundar conteúdos, para tanto precisa conhecer, compreender e apropriar-se a partir do acesso.

Nesse contexto, a utilização do Scratch favorece resultados positivos quando o professor planeja suas ações, considerando as especificidades da sua turma e os objetivos que almeja alcançar. Para isso, faz-se necessário que o professor conheça as potencialidades e os limites dessa linguagem de programação.

\section{Desenvolvimento do trabalho}

O cenário de investigação teve como base de análise uma ação desenvolvida por um professor em readaptação de função que atua como Apoio Pedagógico aos Projetos Tecnológicos desenvolvidos na escola em parceria com o professor da disciplina de matemática.

Destacam-se nesse trabalho, 36 alunos do $6^{\circ}$ Ano de uma Unidade Escolar da rede pública de ensino de Fortaleza. Sendo 9 desses alunos participantes do Projeto Aluno Monitor do Laboratório de Informática Educativa (LIE) desenvolvido pelo professor de Apoio pedagógico aos projetos de tecnologia da escola desde 2016. A referida escola, localiza-se em uma área carente da cidade, atendendo alunos do Ensino Fundamental I até o $6^{\circ}$ Ano.

Os alunos participantes do referido projeto cumpre carga horária de 3 horas semanais, às sextas-feiras. Para participar o discente precisa estar cursando o $6^{\circ}$ Ano. Nesse dia de atendimento os participantes do Projeto de Monitoria recebem aulas de robótica, trabalham com kit Arduino (do professor do LIE) kit LEGO (da escola), e 
sucata eletrônica (recebidos de doação). Esse grupo tem o objetivo de conhecer e usar as tecnologias digitais que podem ser utilizadas no processo de ensino-aprendizagem e trabalhar a programação do Arduino com o Scratch, por ser lúdico e de fácil aprendizado. Também são capacitados para auxiliar os professores e alunos no desenvolvimento das atividades no LIE.

Sobre a participação dos alunos no Projeto de Monitoria, o professor regente de Matemática afirma que:

Notei que os alunos participantes do Projeto de Monitoria, têm apresentado atitudes mais maduras e responsáveis em sala de aula. Eles demonstram mais interesse, concentração e participação em minhas aulas. Inclusive aqueles que apresentavam problemas de indisciplina, atualmente colaboram e gostam de trabalhar em equipe chegando inclusive a elevar os índices de aprendizagem em Matemática. (Professor A).

O Projeto de Monitoria vem colaborando para a melhoria do aprendizado do aluno. Os encontros acontecem no contra turno em um dia da semana caracterizando-se num momento rico para os trabalhos em grupo, ampliando os domínios de compreensão e manipulação dos conceitos de matemática e programação.

Diante desses resultados, resolvemos ampliar as atividades para as turmas regulares, buscando verificar se a motivação dos alunos pelas aulas seria potencializada através de uma proposta de construção de um cubo de LED, utilizando placa Arduino e programando com Scratch. Para executar essa proposta, dividimos a atividade em duas etapas.

A primeira etapa envolveu os alunos na sala de aula do $6^{\circ}$ Ano. Esses alunos detinham um conhecimento inicial sobre lógica para programação, desse modo, foi dispensável as explicações sobre algoritmos.

Entendemos conforme ressalta Rodrigues (2015, p. 64), que o Scratch é uma linguagem de programação "desenvolvida com o objetivo de possibilitar que iniciantes possam criar programas de computador sem aprender a sintaxe de uma linguagem de programação". Com isso, nossa intenção foi motivar o aprendizado dos conceitos de geometria, tema da aula de Matemática, envolvendo os alunos numa experiência divertida por meio da programação.

Os alunos no Laboratório de Informática Educativa (LIE) receberam informações sobre programação com o Scratch, posteriormente às aulas teóricas de matemática sobre figuras geométricas. Um ponto que chamou atenção em todas as aulas foi a concentração dos alunos.

No dia de atendimento no LIE dos alunos do $6^{\circ}$ foi sugerida uma atividade utilizando o Scratch relacionada ao conteúdo de Geometria, que era criar um jogo com dois personagens para dialogarem entre si com perguntas e respostas, gerando desenhos sobre as formas geométricas e suas propriedades. Foi apresentado o Scratch, para que todos pudessem visualizar e acompanhar a aula com os comandos básicos. Cada aluno fez seu jogo individualmente com apoio dos Monitores. Foi observado que o aluno demonstrou interesse em personalizar seu trabalho e realizar as modificações necessárias. A aula tornou-se mais divertida e rica através da troca de experiência. 
Para os Alunos Monitores foi dada uma introdução que abordou os principais aspectos observados na linguagem de programação Scratch como possibilidade para criação das figuras geométricas, especificamente, um cubo de LED. A combinação dos blocos de programação do Scratch facilita a manipulação para dos alunos principiantes. A figura 2, a seguir, demonstra o grupo de alunos monitores montando o cubo.

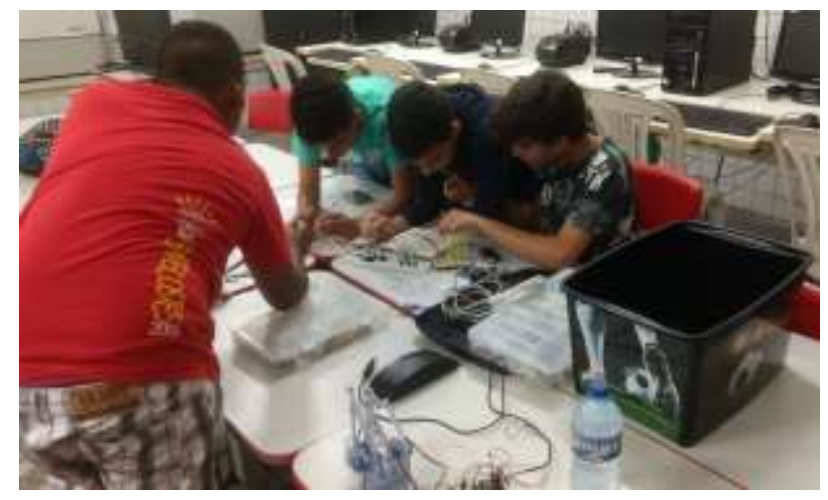

Figura 1. Construção do Cubo de Led pelos monitores

Os nove discentes dividiram-se nas tarefas de acordo com as suas habilidades, demonstrando o potencial do trabalho em grupo. Montar o cubo, testar os LED, e realizar a programação, foram habilidades distintas, onde os alunos envolveram-se mais intensamente de acordo com as suas habilidades. Houve uma distribuição entre os participantes para as seguintes tarefas: $1^{\circ}$ Grupo - Separação e seleção dos materiais: $2^{\circ}$ Grupo - Participaram da montagem, realizaram as ligações dos leds e montaram no gabarito colando com cola quente. Este processo foi feito quatro vezes, pois o cubo tem quatro camadas. E para o $3^{\circ}$ grupo - fazer as ligações no Arduino nas portas digitais, na protoboard e programação.

Os alunos montaram o cubo e realizaram os testes na programação com o Scratch2 utilizando as portas digitais entre 2 e 10.

Com a construção do cubo, os alunos tiveram a oportunidade de visualizar o cubo em três dimensões, além de poder controlar o seu funcionamento, o qual encantou os alunos devido o efeito que consideraram muito bonito. Os alunos fizeram a programação, não sendo possível construir um cubo para cada aluno devido problemas financeiros para aquisição de material para todos os grupos. Mesmo assim, todos participaram criando uma programação para ser testada no cubo. $\mathrm{O}$ efeito de animação no cubo feita por cada grupo foi demonstrado no final da aula.

Em relação ao trabalho de geometria, os alunos realizaram ligações distintas determinando se seriam acesos os LEDs de uma única face, somente dos vértices ou do cubo completo. Essa atividade favoreceu a compreensão das partes de um cubo e dos conceitos específicos da geometria na prática.

Os alunos demonstraram um bom conhecimento de eletrônica, conseguindo identificar os polos positivos e negativos e tomando o devido cuidado para não queimar os componentes. Conseguiram reconhecer as portas digitais do Arduino. A montagem com robótica do cubo de LED será exibida na feira de Ciências da escola, os alunos estão ansiosos para demonstrar o que aprenderam.

A avaliação do trabalho desenvolvido aconteceu de duas formas distintas. Primeiramente, verificando os conhecimentos prévios dos alunos relacionados ao 
VI Congresso Brasileiro de Informática na Educação (CBIE 2017)

Anais do XXIII Workshop de Informática na Escola (WIE 2017)

Scratch de modo a verificar o que eles já sabiam e o que precisavam avançar. Após as atividades foram aplicados um questionário (Formulário Google Drive) e aplicação de Rubricas Avaliação, de modo a engajar os alunos nas tarefas que deveriam realizar, para conhecimento desde o princípio o que era esperado de cada um dos alunos.

A seguir, apresentamos as informações obtidas nessas avaliações.

\section{Avaliação diagnóstica e uso de rubricas}

Para conhecer a opinião dos alunos do Ensino Fundamental II, sobre a facilidade e/ou complexidade relacionada a utilização do Scratch para aprender conteúdos de matemática, enviamos um questionário on line (Google Drive), com questionamentos referentes ao uso que os alunos já faziam do Scratch. Tivemos o retorno de 36 alunos, os quais responderam o questionário, sendo 26 do sexo feminino e 10 do sexo masculino, com idades compreendidas entre 11 a 16 anos.

A seguir as percepções mais relevantes dos alunos sobre o uso do Scratch:

Tabela 1. Facilidade de Uso

\begin{tabular}{|l|c|c|c|c|c|c|}
\hline \multirow{2}{*}{ Itens } & \multicolumn{2}{|c|}{ Discordo } & \multicolumn{2}{c|}{ Indeciso } & \multicolumn{2}{c|}{ Concordo } \\
\cline { 2 - 7 } & T.A & \% & T.A & \% & T.A & $\%$ \\
\hline $\begin{array}{l}\text { É fácil programar com } \\
\text { Scratch. }\end{array}$ & 0 & 0 & 3 & 8,3 & 33 & 91,7 \\
\hline $\begin{array}{l}\text { É divertido programar com } \\
\text { Scratch. }\end{array}$ & 0 & 0 & 0 & 0 & 36 & 100 \\
\hline $\begin{array}{l}\text { É simples acessar o Scratch } \\
\text { no computador }\end{array}$ & 0 & 0 & 2 & 5,6 & 34 & 94,4 \\
\hline
\end{tabular}

A maioria dos alunos $(91,7 \%)$ considera que é fácil usar o Scratch, demonstrando que os alunos já têm propriedade para acessar e desenvolver a atividade sugerida. Destaca-se também que todos os alunos da turma (100\%) foram unânimes ao considerar que programar no Scratch é divertido e apresenta forma simples para acesso pelo computador.

Tabela 2. O Scratch como recurso para a aprendizagem

\begin{tabular}{|l|c|c|c|c|c|c|}
\hline \multicolumn{1}{|c|}{ Itens } & \multicolumn{2}{|c|}{ Discordo } & \multicolumn{2}{c|}{ Indeciso } & \multicolumn{2}{c|}{ Concordo } \\
\cline { 2 - 7 } & T.A & $\%$ & T.A & $\%$ & T.A & \% \\
\hline $\begin{array}{l}\text { Envolvi-me nos assuntos de matemática } \\
\text { enquanto jogava }\end{array}$ & 0 & 0 & 0 & 0 & 36 & 100 \\
\hline $\begin{array}{l}\text { Aprendo mais Matemática quando jogo } \\
\text { Scratch }\end{array}$ & 0 & 0 & 0 & 0 & 36 & 100 \\
\hline $\begin{array}{l}\text { Fico mais concentrado no assunto quando } \\
\text { jogo como Scratch }\end{array}$ & 0 & 0 & 3 & 8,3 & 33 & 91,7 \\
\hline $\begin{array}{l}\text { Gosto quando o professor do LIE explica a } \\
\text { matéria de matemática utilizando o Scratch }\end{array}$ & 0 & 0 & 0 & 0 & 36 & 100 \\
\hline
\end{tabular}

Relativamente à percepção dos alunos sobre se o Scratch pode ser considerado um recurso de apoio à aprendizagem (ver Tabela 2), todos os alunos (100\%) 
VI Congresso Brasileiro de Informática na Educação (CBIE 2017)

Anais do XXIII Workshop de Informática na Escola (WIE 2017)

concordaram que se envolvem nos assuntos de matemática enquanto jogam resultando numa maior aprendizagem. Foi constatado que $(91,7 \%)$ demonstrou maior atenção no assunto de matemática quando jogava com o Scratch. E por fim os alunos (100\%) relataram que gostam quando o professor do LIE explica a matéria de matemática utilizando o Scratch.

Em relação as rubricas ${ }^{1}$, segundo o prof. do LIE, essa estratégia possibilitou:

Uma forma nova de avaliação, facilitando a organização e a coleta dos dados. Os mesmos ficam registrados como forma de pensamento organizado para facilitar a compreensão do processo de aprendizagem dos alunos. (Professor B).

Durante a aplicação das rubricas para o grupo de alunos monitores não tiveram problema para resolver, foram sinceros nas respostas, ficavam comparando as respostas e discutindo quem tinha realizado mais atividades ou participado mais.

A seguir alguns resultados relevantes da rubrica sobre a avaliação do trabalho em grupo:

Tabela 3. Rubrica: Avaliação do Trabalho em Grupo

\begin{tabular}{|l|c|c|c|c|}
\hline \multicolumn{1}{|c|}{ CRITÉRIOS } & $\begin{array}{c}\text { NÃO ESTOU } \\
\text { LIGANDO }\end{array}$ & $\begin{array}{c}\text { TANTO } \\
\text { FAZ }\end{array}$ & $\begin{array}{c}\text { ESFORCEI } \\
\text { BASTANTE }\end{array}$ & ARRASEI \\
\hline Contribuições & - & 1 & 8 & - \\
\hline Resolução de Problemas & - & 2 & 3 & 4 \\
\hline Foco na Tarefa & - & 2 & 6 & 1 \\
\hline Desenvolvimento do Trabalho em Equipe & 2 & 1 & 5 & 1 \\
\hline
\end{tabular}

Na Tabela 3, observa-se que os 9 alunos participantes do Projeto de Monitoria, informaram que contribuíam rotineiramente com boas ideias nas discussões em grupo e atuavam como um líder que contribui com muito esforço. Sobre a resolução de problemas 4 alunos afirmaram que buscavam e sugeriam soluções ativamente para a soluções de problemas. Quanto ao foco na tarefa, 6 estudantes responderam que na maior parte do tempo focavam na tarefa e no que precisava ser feito e que os demais membros do grupo puderam contar uns com os outros. Sobre o desenvolvimento do trabalho em grupo, 5 deles afirmaram que davam ideias, argumentavam e contra argumentavam, melhorando as próprias ideias e as dos colegas, mas não contribuíam para a formação de um ambiente rico com participação de todos.

Tabela 4. Rubrica: Construção e Programação do Cubo

\begin{tabular}{|l|c|c|c|c|}
\hline \multicolumn{1}{|c|}{ CRITÉRIOS } & $\begin{array}{c}\text { NÃO ESTOU } \\
\text { LIGANDO }\end{array}$ & $\begin{array}{c}\text { TANTO } \\
\text { FAZ }\end{array}$ & $\begin{array}{c}\text { ESFORCEI } \\
\text { BASTANTE }\end{array}$ & ARRASEI \\
\hline Planejamento do Projeto & 1 & -- & 4 & 4 \\
\hline Desenvolvimento do Projeto & -- & 1 & 4 & 3 \\
\hline Programação & 1 & 5 & 3 & -- \\
\hline Documentação & -- & 1 & 6 & 1 \\
\hline
\end{tabular}

$\mathrm{Na}$ tabela 4, podemos observar a avaliação dos alunos monitores sobre construção e programação do Cubo no quesito "Graduação de Qualidade": Sobre o

${ }^{1}$ Para saber mais basta acessar https://goo.gl/Sc2JBv Acesso Maio de 2017. 
Planejamento do projeto, 4 desses alunos se esforçaram e arrasaram na elaboração e execução das etapas do planejamento. Para o desenvolvimento e protótipo do cubo, 4 alunos se esforçaram bastante, colocando a mão na massa. Para a programação em si, 5 alunos, deram algumas ideias para programar. Para os registros como documentação, 6 alunos se esforçaram bastante e contribuíram com alguns materiais significativos para 0 registro do processo do trabalho.

\section{Resultados e Discussões}

Tendo em vista o desenvolvimento do Projeto Aluno Monitor sobre programação, podemos ressaltar a mudança de comportamento dos alunos monitores através da elevação da autoestima resultando na melhoria do relacionamento entre eles, do desenvolvimento do trabalho em equipe, do respeito ao outro e isso faz muita diferença na vida deles.

Os resultados apontaram que, após as aulas de robótica aplicada foi possível detectar que os alunos realizaram com sucesso a criação do cubo de LED demonstrando o interesse e o aprendizado dos conceitos de geometria utilizando a linguagem de programação Scratch e o Arduino. Os estudantes acompanharam com facilidade todo o processo para o desenvolvimento do trabalho no decorrer da aula.

$\mathrm{Na}$ Matemática aprenderam de forma lúdica as formas geométricas planas e espaciais, aresta, face, vértice, ponto, reta. Este cubo pode ser aproveitado em outras aulas, como número quadrado perfeito, potenciação, resultando em aulas mais práticas.

Acreditamos no potencial desses alunos que fazem parte do Projeto de Monitoria. As atividades desenvolvidas por eles na robótica repercutem na comunidade escolar, surpreendendo outros professores e os demais colegas, que inclusive estão querendo participar do Projeto. A ampliação da atividade desenvolvida com os monitores para as turmas regulares favoreceu um aprendizado mais significativo e motivante para os demais alunos.

\section{Considerações finais e trabalhos futuros}

Esse trabalho apresentou uma análise dos resultados de uma prática pedagógica desenvolvida com um grupo de alunos do $6^{\circ}$ ano $\mathrm{C}$ e um grupo de Alunos Monitores, utilizando o Scratch e o Arduino para programar um cubo LED visando analisar a influência no uso de programação no desenvolvimento do raciocínio lógico e na elaboração de conteúdos de geometria. Onde os alunos construíram e programaram um cubo por meio do encadeamento de blocos de comandos.

Verificamos um maior interesse dos alunos na aula de programação e montagem do cubo, auxiliando na participação e compreensão dos alunos, por estarem interessados e atentos aos conceitos que estavam sendo desenvolvidos, dando indício de melhoria na aprendizagem. No entanto, não tivemos dados suficientes para analisar o verdadeiro resultado do impacto na aprendizagem dos alunos através dessa estratégia, sendo uma necessidade para um trabalho futuro.

\section{Referências}

Amorim, J. C; Silva, N. C.; Santos, M. S.; Silva, F. L. Integrando as Plataformas App Inventor e Arduino na Construção de um Humanoide. Anais do Workshop de 
VI Congresso Brasileiro de Informática na Educação (CBIE 2017)

Anais do XXIII Workshop de Informática na Escola (WIE 2017)

Informática na Escola, WIE 2016. Disponível em <http://brie.org/pub/index.php/wie/article/view/6622/4533> Acesso em jun. 2016.

Albuquerque, D; Bremgartner, V; Lima, H; Salgado, N. Uma Experiência do Uso Do Hardware Livre Arduino no Ensino De Programação De Computadores. Anais do Workshop de Informática na Escola, WIE 2016. Disponível em <http://brie.org/pub/index.php/wie/article/view/6602/4513> Acesso em jun. 2016.

Brasil. Secretaria de Educação Fundamental. Parâmetros Curriculares Nacionais: Matemática $-5^{\mathrm{a}}$ a $8^{\mathrm{a}}$ série. Brasília: MEC/SEF, 1998. Disponível em $<$ http://portal.mec.gov.br/seb/arquivos/pdf/introducao.pdf $>$ Acesso em abr. 2016

Buckingham, D.; BURN, A. Game literacy in theory and practice. In: Journal of Educational Multimedia and Hypermedia: Association for the Advancement of Computing in Education. AACE, Norfolk, n.16, v.3, p.323-349, 2007.

Papert, S. Logo: Computadores e educação. São Paulo: Editora Brasiliense, 1985.

Rodrigues, C. L.; ZEM-Lopes, M. ; Marques, L.; Iotani, S. Pensamento Computacional: transformando ideias em jogos digitais usando o Scratch. In Anais do XXI Workshop de Informática na Escola, WIE 2015. Disponível em <http://www.br-ie.org/pub/index.php/wie/article/view/4992> Acesso em mar. 2016.

Sobreira, e. S. R.; Viveiro, A. A; D'Abreu, J. V. V. Do Paper Circuit à programação de Arduino com Scratch: uma sequência didática para aprendizagem do conteúdo de energia nos anos iniciais do ensino fundamental. Anais do Workshop de Informática na Escola, WIE 2016. Disponível em $<$ http://www.brie.org/pub/index.php/wie/article/view/6852/4730> Acesso em jun. 2016.

Zanetti, H. A. P.; OLIVEIRA, C. L. V. Prática de ensino de Programação de Computadores com Robótica Pedagógica e aplicação de Pensamento Computacional. In Anais dos Workshops do IV Congresso Brasileiro de Informática na Educação, CBIE 2015. Disponível em $<$ http://www.brie.org/pub/index.php/wcbie/article/view/6268> Acesso em jan. 2017. 